\title{
Study on an Interactive Roaming Control Scheme for Industrial Products Based on Virtual Reality Technology
}

\author{
Yimin Su \\ College of art and design, Beijing University of Technology, Beijing 100029, P.R. China. \\ suyimin@yeah.net
}

Key words: Virtual reality technology, Industrial design, Roaming control

\begin{abstract}
In this paper, it develops a set of interactive roaming control scheme applied to the virtual reality system through the research and study on human engineering characteristics. This scheme realized the functions of viewpoint rotation, visual angle change and scale transformation in the virtual scene, which can verify the feasibility of this method through the development of the navigation map.
\end{abstract}

\section{Introduction}

Virtual reality technology is a very active area of research at present, which is also a collection of a series of high technology both in science field and technology field, which can be including information engineering, multimedia technology, artificial intelligence, graphics, human engineering technology, sensor technology and parallel computing technology. In addition, it also includes the study on the science of human behavior and so on[1]. Virtual reality is a higher level of the development of multimedia technology, integrating and penetrating more of other high level technologies. It can provide a more immersive experience for the user, which can be regarded as the window for people to explore the microscopic world and macroscopic world, which can provide the method to make research on the essence and mechanism of things for people that can not be proximity to the scene or who can not have directly observation on the microstructure. In the virtual reality environment, the first experience of the user is "immersion", or the feeling that is generated in the computer world. It also can interact with the environment through its own consciousness. An combination between immersive feeling and interaction can be called as telepresence. Computer scientist Jonathan defined it as " in some extent, it is felt in the mediated environment, rather than directly in the physical environment."In other words, an effective VR experience can make you unaware of the true environment and focus on your existence in the virtual environment[2-3].

In this paper, it has developed a set of virtual scene roaming control scheme based on the combination of computer users' habit and human engineering characteristics, at the same time, this scheme has been verified by the development of navigation map.

\section{The Roaming Control Scheme of Scene}

In $V C++$ and $C++$ windows programming, there is a special message control mechanism, which can easily capture the keyboard and mouse operation, in addition, in the OpenGL function library, it also has the corresponding capture function. In this paper, it can use GLUT utility library to capture the corresponding message.

Such as: the function of capturing the mouse is: glutMouseFunc(mouse-porc);

The function of capturing keyboard is: glutKeyboardFunc (key);

Among them, mouse-proc, key, special is the function name of the corresponding application.

In this system the key on the keyboard keys and "-" number, "=" to control forward, backward, leftward and rightward and acceleration, deceleration, etc., users, and the scene of the interactive action, the redrawing of the scene. Complete the redrawing of the scene according to the user's actions, there are two ways: one is accomplished by directly changing the world coordinate; the 
second is to transform the viewpoint to complete interactive scene change with the aid of the. The direct change of the world coordinates will involve the transformation of the coordinates of all the objects in the scene, and the error accumulation can be caused by many times, which will result in the distortion of the scene. Therefore, in the construction of interactive real time virtual scene, it should be through the transformation of the user viewpoint to complete the control of the scene.

There is a dedicated fuction in the GLU utility library, namely, gluLookAt(ex, ey, ez, cx, $c y, c z, u x, u y, u z)$, among them, ex, ey, ez is theEZ for the 3D coordinates of the human eye, $c x, c y$ and $c Z$ is the 3D coordinates of the reference point, UX, uy, UZ is the upside vector. This function can be easily realized by using it. The function gluLookAt is actually a series of translation and rotation commands, which generates a view matrix derived from the viewpoint position, the reference point in the center of the scene, as well as the vector. This matrix converts the viewpoint to the origin and converts the reference point to the negative $Z$ axis. Point of view and reference point can be connected in view, forming the observation direction, while the upside vector can point out the direction of the view from bottom to top, usually the upside vector can be replaced to the positive $Y$ axis, which is not necessarily paralleled on the line from the view point to the reference line.

In the virtual construction roaming, due to the standing direction of people is always upside, the coordinate vector $(u x, u y, u z)$ is fixed as $(0 f, 1.0 f, 0.0 f)$, in this way, it can capture the corresponding operation of the users according to the keyboard and mouse, therefore, through the related mouse displacement or the operation of keyboard (if it is through the operation of the keyboard,it can define and adjust the speed in advance through a variable), it can calculate the changes in the human eye and a point of reference (using three-dimensional geometric knowledge), then it can automatically generate the corresponding 3D scene through gluLookAt(ex, ey,ez, cx, $c y, c z, u x, u y, o z)$. For example, the related formula of making left turn (using pseudo code) as follows:

$$
\begin{aligned}
& c x=(c x-e x) * \cos A+(c z-e z) * \sin A+e x ; \\
& c z=(c z-e z) * \cos A-(c x-e x) * \sin A+e z ;
\end{aligned}
$$

Among them, A is the step size of a rotation angle that is defined by system, if it turns right, then A can be changed into -A. Forward is as follows:

$$
\begin{gathered}
a=\operatorname{sqrt}((c x-e x) *(c x-e x)+(c z-e z) *(c z \cdot e z)) ; \\
e x=\left(\text { speed }^{*}(c x-e x)+a^{*} e x\right) / a ; \\
e z=\left(\operatorname{speed}^{*}(c z-e z)+a^{*} e z\right) / a ; \\
c x=\left(\operatorname{speed}^{*}(c x-e x)+a^{*} c x\right) / a ; \\
c z=\left(\operatorname{speed}^{*}(c z-e z)+a^{*} c z\right) / a ;
\end{gathered}
$$

Among them, speed is the step size of the forwards. If it is backwards, then speed can be changed into -speed.

\section{The Interaction Mode of Scene}

Interactive roaming of the scene is the control method that users can control the position and direction of roaming in accordance with their intention through using keyboard, mouse or joystick and some other inputting devices.

The browsing processof users is actually a 3D imaging process by having constant move and change in point of view and the direction of the view, the direction of gaze can be determined by our observation point, system can browse different 3D effect through the constant change in viewpoint and viewing position. Browsing forwards, backwards, making the direction of rotation, looking up and looking down, besides, it also can replace the changes in the observation point 
through the move of mouse.After the system obtained the keyboard command or the mouse position data, it can change the corresponding parameters and re-draw the scene.

The interaction mode of scene in this system (see Table 1):

Table1 Table scene interactively

\begin{tabular}{|c|c|c|c|}
\hline Control name & English name & Operation mode & $\begin{array}{l}\text { Change of the } \\
\text { visual angle }\end{array}$ \\
\hline $\begin{array}{l}\text { Left and right } \\
\text { rotation }\end{array}$ & Apoint_L/R & $\begin{array}{l}\text { Press the left mouse button } \\
\text { and drag at the same time } \\
\text { (onMouseL+Drag) }\end{array}$ & $\begin{array}{c}\text { Rotating the } \\
\text { direction of the } \\
\text { vision as the same } \\
\text { as the } \\
\text { displacement } \\
\text { direction of the } \\
\text { mouse }\end{array}$ \\
\hline $\begin{array}{l}\text { Zoom the angle } \\
\text { of the view }\end{array}$ & $\begin{array}{c}\text { Apoint_View_ } \\
\text { ZP/ZC }\end{array}$ & $\begin{array}{l}\text { Scroll the middle of the } \\
\text { wheel of the mouse } \\
\text { (onMouseCenRun) }\end{array}$ & $\begin{array}{l}\text { Adjust the radius } \\
\text { of observation, to } \\
\text { achieve zoom }\end{array}$ \\
\hline $\begin{array}{l}\text { Adjustment of } \\
\text { elevation }\end{array}$ & $\begin{array}{c}\text { Apoint_Elevation_ } \\
\text { T/D }\end{array}$ & $\begin{array}{l}\text { Press the up key or down } \\
\text { key of the } \\
\text { keyboard(KeyboardTop/D } \\
\text { own) }\end{array}$ & $\begin{array}{l}\text { Adjust the } \\
\text { elevation angle of } \\
\text { the observation } \\
\text { point, achieving } \\
\text { the effect of } \\
\text { looking up to the } \\
\text { upside and looking } \\
\text { down to the } \\
\text { downside }\end{array}$ \\
\hline $\begin{array}{l}\text { Change the } \\
\text { point of } \\
\text { observation }\end{array}$ & Apoint_Move & $\begin{array}{l}\text { Press the left key or right } \\
\text { key of the } \\
\text { keyboard(KeyboardLeft/R } \\
\text { ight) }\end{array}$ & $\begin{array}{l}\text { Change the } \\
\text { position of the } \\
\text { observation point, } \\
\text { but facing the } \\
\text { same direction. }\end{array}$ \\
\hline
\end{tabular}

\section{Rotation of the Viewpoint}

Establishing three-dimensional coordinates, namely, $W x, W y$ and $W z$, defining the radius of world hemisphere is $r$, in the coordinate system, setting the initial observation sites as Apoint, establishing the Apoint as the origin point, paralleled to the local coordinate system of the world coordinate system, namely, Ex、Ey、Ez, when the viewpoint is controlled and rotated by the mouse, it can get the displacement coordinate of the mouse:

$$
X=X+X^{\prime} ; Y=Y+Y^{\prime}
$$

After $X, Y$ finished the displacement, it can get the coordinate system aftter finishing rotation angle:

$$
E x=(\cos X, 0,-\sin X) ; E z=\text { Apoint-Apoint'; Ey=Ex*Ez; }
$$

The global coordinates in the world coordinate system should be as follows:

Apoint $x=$ Apoint. $x+r \cos Y^{*} \sin X ;$ Apoint' $z=$ Apoint. $z+r \cos Y^{*} \cos X ;$ Apoint' $y=$ Apoint. $y+r \cos Y$

Though the above formula, we can calculate the angle of the view during the mouse had rotation operation.

\section{Change of the Visual Angle}

Changes in the visual angle can be finished by the control of the up key and down key, left key and right key of the keyboard, defining the temporary observation reference point as Bpoint, the $X$ direction of viewpoint Apoint can not be changed, $Z$ can change to $Z^{\prime}$, which can cause $Y$ change to $Y^{\prime}$, therefore, it can get:

$$
\text { Bpoint.y }=\text { Apoint. } y+(-) d \text {; }
$$

Among them, $d$ is the length of step which is a constant.

$$
X^{\prime}=X ; Z^{\prime}=\text { Bpoint-Apoint; } Y^{\prime}=X^{\prime *} Z^{\prime}
$$

Through the above formula, we can calculate out the position of view coordinates after changing the viewpoint. 


\section{Change of Zoom}

Unlike the changes in zoom of the model the zoom of view that is based on view coordinate can be changed by the method of changing the distance between the viewpoint and the reference point of observation, defining $r^{\prime}$ as the radius of the new hemisphere: $r^{\prime}=r \pm d, d$ is the number of squares that the rolling wheel of mouse rolled, generally speaking, it is usually defined between $(0, r / 3)$, while the direction of the $\mathrm{X}, Y$ view coordinate system does not change when it zooms.

\section{The Design and Fabrication of Navigation Map}

As for the set of interface, it can use GUI of the engine system, in the engine system, it can use the interface components of Windows to build 3D program, then setting the interface into the 3D scene, so as to make the interface integrated with 3D scene, making the operation more intuitive and convenient. The navigation map in the virtual scene can make the map interface be fabricated into the scene, so as to realize the location of the user's scene. GUI (Graphical User Interface) can use the mouse to click on the window button to operate, which is like the operation of Windows that can use graphical interface to operate, both of which are very intuitive.The setting of GUI can reduce the user's cognitive burden, which can maintain the consistency of the interface, at the same time, it can build up the interactive communication between the interface with the user.This kind of customer-oriented design makes the operation more humane, which can reduce the user's cognitive burden, so that it is more suitable for the user's operational requirements.GUI can have computer interface operation through using windows, menus, buttons, and other graphical methods, which can be convenient for the use of non-professional users.Interface design for Yellow River Museum can set the window of electronic map navigation through the engine of GUI. First of all, by means of using Photoshop, it can design and fabricate the planar graph of the electronic map.Then it can edit the set of map position by means of using QDE (the third party software) to edit, the edition of the script can contain the name of map,the position of the mapand so on. In the QDE script editor, the window of navigation map can be written as follows:

Because GUI is embedded in the 3D scene, so the graphic interface background of GUI can be partly transparent or fully transparent, through such a transparent effect processing, GUI graphical interface can be integrated with 3D background, thus, in vision, it is more natural. At the same time, it does not affect the roaming visual in 3D scene .

\section{Conclusion}

In this paper, a display of roaming control scheme based on virtual reality technology products has been studied in detail, this kind of scheme used ergonomic principles that can provide a convenient and useful solution by using a keyboard, a mouse, so as to observe the virtual scene for the viewers. This method is applicable to all kinds of virtual reality display systems, which can provide a new way to control the scene of virtual reality.

\section{Reference}

[1] Xu, Pei Long, and Yi Min Su. "Design and Implementation of landscape system for East and West Huashi Street in Beijing based on virtual reality technology." Applied Mechanics and Materials. Vol. 263. Trans Tech Publications, 2012.

[2] Xin, Lan, and Lan Chengrong. "Quantum-Brownian Motion and Brownian Bridge Path Integral of a Free Particle." Journal of Applied Science and Engineering Innovation 2.7 (2015): 261-266.

[3] Wang, Xuesong, Mingquan Zhou, and Luan Hua. "Virtual Learning 3D Environment on Cloud Service Platform." Journal of Applied Science and Engineering Innovation Vol 1.3 (2014). 